\title{
Transcriptional regulation of $\alpha$-synuclein: insights from blood?
}

\section{Marie-Francoise Chesselet ${ }^{\dagger} \&$ Beate Ritz}

${ }^{\dagger}$ Author for correspondence: Department of Neurology, David Geffen School of Medicine at UCLA, 710 Westwood Plaza, Los Angeles, CA 90095, USA = Tel.: +1 3102671781 = Fax: +1 3102671786 nnchessel@ucla.edu

Evaluation of: Scherzer CR, Grass JA, Liao $Z$ et al.: GATA transcription factors directly regulate the Parkinson's disease-linked gene $\alpha$-synuclein. Proc. Natl Acad. Sci. USA 105(31), 10907-10912 (2008). Based on genetic and pathological information, the vesicular protein $\alpha$-synuclein has recently emerged as one of the key proteins involved in Parkinson's disease (PD). In elegant studies in blood and nigrostriatal dopaminergic neurons, Scherzer et al. identified novel mechanisms that regulate $\alpha$-synuclein expression, and suggest the possibility of coregulation of $\alpha$-synuclein levels, a likely contributor to PD risk, and iron, which has also been implicated in the pathophysiology of the disorder. These data point to a new potential molecular target for delaying PD onset or progression.

\section{Summary of methods \& results}

Scherzer et al. took the unusual and creative approach to focus on the mechanisms regulating $\alpha$-synuclein levels in blood, where they found the protein to be highly expressed in red blood cells [1]. This gave them access to a large number of human samples, which is generally not possible with most other tissue, in particular brain. Using an initial sample of 22 control subjects, they searched for genes that were tightly correlated with $\alpha$-synuclein $(S N C A)$ gene expression and then confirmed the positive association in additional cohorts totaling 89 healthy individuals, concluding that the coexpression of the genes identified is robust and a biologically relevant finding. Of the associated genes ( $A L A S 2$, FECH and $B L V R B), A L A S 2$ is known to be regulated by the transcription factor GATA1, thus indicating this factor as a potential regulator of $S N C A$ expression. Using genetic complementation analysis in GATA1-null erythropoid precursor cells, the authors identified a GATA-binding site in intron 1 of the SNCA gene, supporting the possible role of the GATA1 transcription factor in the regulation of SNCA expression.

While these data are exciting, they do not necessarily account for the regulation of $\alpha$-synuclein in neurons, where the protein is probably causing some of the Parkinson's disease (PD) pathology, because GATA1 is not expressed in neuronal cells. The authors thus turned their attention to GATA2, which has overlapping activities with GATA1 in the control of embryonic erythrogenesis. First, they demonstrated that GATA2 is expressed in dopaminergic neurons, a primary site of $\alpha$-synuclein accumulation and cell death in $\mathrm{PD}$, and that it binds to the same intronic site in the SNCA gene as GATA1 did in the blood cell experiments. Furthermore, knocking down GATA2 in a dopaminergic cell line in fact reduced $\alpha$-synuclein mRNA and protein levels.

\section{Discussion}

Since the identification 10 years ago of rare mutations in two families with hereditary PD, the role of $\alpha$-synuclein in PD has been extensively researched [2]. Indeed, although mutations in $\alpha$-synuclein are exceedingly rare, abnormal accumulation of $\alpha$-synuclein occurs in the brain of patients with the sporadic form of the disease. Further supporting a causal role of increased $\alpha$-synuclein levels in PD, some familial forms of the disease are due to multiplication of the $S N C A$ gene. Furthermore, polymorphisms that may alter $\alpha$-synuclein levels may also alter the risks of developing sporadic PD [3]. A re-evaluation of PD pathology based on immunostaining for $\alpha$-synuclein revealed extensive accumulation of the protein in neuronal cytoplasm (in the form of proteinaceous inclusions - the Lewy bodies) and processes [4]. This $\alpha$-synuclein pathology appears to progress in well-ordered stages, starting in the medulla (stage 1), and progressing to the locus coeruleus, raphe nuclei, reticular formation (stage 2), pedunculopontine nucleus, substantia nigra, amygdala (stage 3), thalamus (stage 4) and finally cerebral cortex (stage 5). When $\alpha$-synuclein pathology reaches the substantia nigra it leads to the progressive loss of dopaminergic neurons and the defining motor symptoms of PD [5].

It is not known whether $\alpha$-synuclein accumulation in neurons of patients with sporadic forms of the disease is due to increased levels of the protein, abnormal conformation resulting
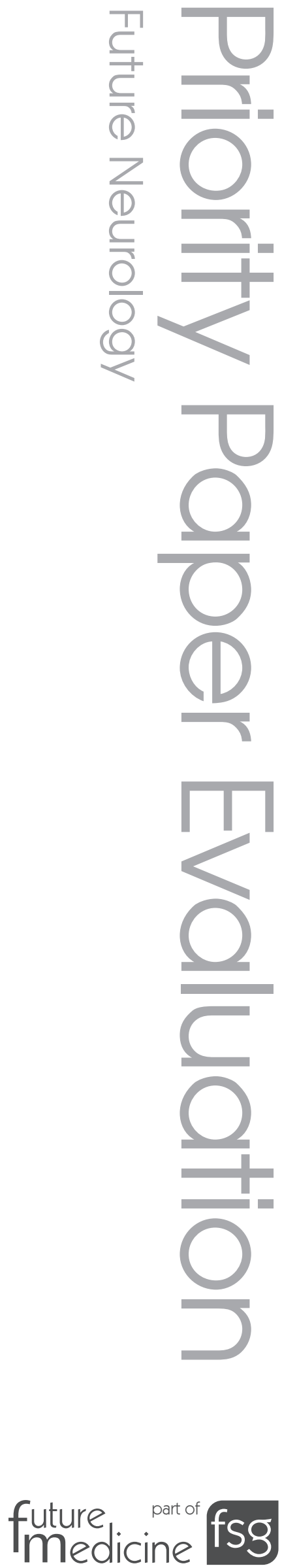
from modifications induced by oxidative stress, or abnormal cleavage [6]. The genetic evidence points to a role for increased levels of $\alpha$-synuclein as a key pathological factor in PD, suggesting that dysregulation of molecular mechanisms controlling $\alpha$-synuclein levels could contribute to $\mathrm{PD}$, and that reducing $\alpha$-synuclein levels could be beneficial in PD.

Surprisingly little is known about factors regulating the level of expression of $\alpha$-synuclein considering its potential importance for understanding and treating PD. The data presented in this paper are particularly exciting because they identify one potential key regulator of $\alpha$-synuclein levels [1]. In addition, they link the expression of $\alpha$-synuclein to iron and heme metabolism, suggesting a commonality in pathways for two well-known features of PD (i.e., Lewy body formation and iron accumulation) [7].

Iron accumulates in the substantia nigra in PD [8], induces $\alpha$-synuclein fibril formation in vitro, and may contribute to protein aggregation and Lewy body formation through interactions with $\alpha$-synuclein [9]. Furthermore, excess iron is linked to nigrostriatal degeneration in experimental models [10]. Based on the data of Scherzer et al., one could speculate that in neurons, GATA2 may coregulate genes related to $\alpha$-synuclein as well as iron balance in neurons, just like GATA1 does in immature blood cells [1]. One obvious candidate would be ferrochelatase (FECH), which Scherzer demonstrated to be coregulated by GATA1 in blood cells and which is known to localize to the mitochondrion and to catalyze the insertion of the ferrous form of iron into protoporphyrin IX for heme biosynthesis [11]; thus, increased expression of FECH may result in increased heme biosynthesis. Little is known about the consequence of heme increase in brain cells but heme is known to be pro-oxidative in nature [11].

\section{Conclusion}

This manuscript describes a creative approach - the analysis of genes that are coregulated with $\alpha$-synuclein in blood cells - to identify novel mechanisms of transcriptional regulation of $\alpha$-synuclein. Based on the research on peripheral cells, the authors then identified a transcription factor able to regulate $\alpha$-synuclein expression in those neurons that are affected in PD. The research is important since convergent genetic evidence link high levels of expression of $\alpha$-synuclein with the development of PD. The research also identifies a possible link between the regulation of $\alpha$-synuclein expression and genes involved in iron regulation, another factor implicated in PD.

\section{Future perspective}

Genetic and pathological evidence linking $\alpha$-synuclein not only to rare familial forms of PD but also to the much more frequent sporadic forms of the disease continues to accumulate. Mechanisms that lead to the abnormal accumulation of $\alpha$-synuclein in sporadic PD are unknown. Identifying molecular mechanisms that regulate the expression of this protein may point to new pathophysiological mechanisms involved in PD. Whether there is a causal relationship between high levels of $\alpha$-synuclein and sporadic PD remains unresolved. Identifying ways to safely reduce the level of this protein in patients will be necessary to answer this question and will hopefully be of therapeutic value. The data in this study are a first step towards an understanding of $\alpha$-synuclein transcriptional regulation. More research to elucidate its complexity is now warranted and could shed light on the relationship between iron and $\alpha$-synuclein in the pathophysiology of PD.

\section{Financial \& competing interests disclosure \\ The authors have no relevant affiliations or financial involvement with any organization or entity with a financial interest in or financial conflict with the sub- ject matter or materials discussed in the manuscript. This includes employment, consultancies, honoraria, stock ownership or options, expert testimony, grants or patents received or pending, or royalties. \\ No writing assistance was utilized in the production of this manuscript.}

\section{Executive summary}

- Increased expression of $\alpha$-synuclein is linked to Parkinson's disease.

- Using blood samples, Scherzer et al. demonstrated coinduction of the $\alpha$-synuclein (SNCA) gene and three genes involved in heme metabolism.

- The transcription factor GATA1 regulates these genes in blood and binds to one intron of SNCA.

- The transcription factor GATA2 binds to SNCA in dopaminergic neurons and modulates its expression.

- The results suggest that GATA is a novel molecular target to decrease $\alpha$-synuclein production, which could be beneficial in Parkinson's disease. 


\section{Bibliography}

1. Scherzer CR, Grass JA, Liao Z et al:: GATA transcription factors directly regulate the Parkinson's disease-linked gene $\alpha$-synuclein. Proc. Natl Acad. Sci. USA 105(31), 10907-10912 (2008).

2. Klein C, Schlossmacher MG: Parkinson disease, 10 years after its genetic revolution: multiple clues to a complex disorder. Neurology 69(22), 2093-2104 (2007).

3. Ross OA, Gosal D, Stone JT et al:: Familial genes in sporadic disease: common variants of $\alpha$-synuclein gene associate with Parkinson's disease. Mech. Ageing Dev. 128(5-6), 378-382 (2007).

4. Braak H, Del Tredici K: Invited Article: Nervous system pathology in sporadic Parkinson disease. Neurology 70(20), 1916-1925 (2008).

5. Dickson DW, Fujishiro H, DelleDonne A et al:: Evidence that incidental Lewy body disease is pre-symptomatic Parkinson's disease. Acta Neuropathol. 115(4), 437-444 (2008).
6. Waxman EA, Giasson BI: Molecular mechanisms of $\alpha$-synuclein

neurodegeneration. Biochim. Biophys. Acta DOI: 10.1016/j.bbadis.2008.09.013 (2008) (Epub ahead of print).

7. Gotz ME, Double K, Gerlach M, Youdim MB, Riederer P: The relevance of iron in the pathogenesis of Parkinson's disease. Ann. NY Acad. Sci. 1012, 193-208 (2004).

8. Berg D: Disturbance of iron metabolism as a contributing factor to $\mathrm{SN}$ hyperechogenicity in Parkinson's disease: implications for idiopathic and monogenetic forms. Neurochem. Res. 32, 1646-1654 (2007).

9. Uversky VN, Li J, Fink AL: Metal-triggered structural transformations, aggregation, and fibrillation of human $\alpha$-synuclein. A possible molecular NK between Parkinson's disease and heavy metal exposure. J. Biol. Chem. 276, 44284-44296 (2001).

10. Kaur D, Peng J, Chinta SJ et al:: Increased murine neonatal iron intake results in Parkinson-like neurodegeneration with age. Neurobiol. Aging 28(6), 907-913 (2007).
11. Atamna H: Heme, iron, and the mitochondrial decay of ageing. Ageing Res. Rev. 3(3), 303-318 (2004).

\section{Affiliations}

- Marie-Francoise Chesselet Department of Neurology, and,

Department of Neurobiology, David Geffen School of Medicine at UCLA, 710 Westwood Plaza, Los Angeles, CA 90095, USA

Tel.: +1 3102671781

Fax: +1 3102671786

mchessel@ucla.edu

- Beate Ritz

Department of Neurology,

and,

Department of Epidemiology, UCLA, Los Angeles, USA

Tel.: +1 3102067458

Fax: +1 3102066039

britz@ucla.edu 